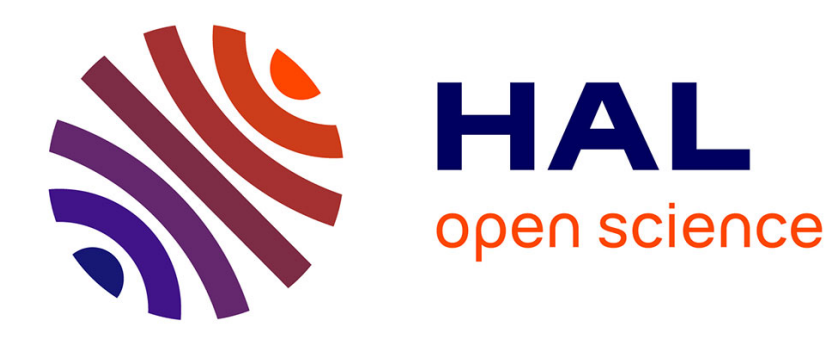

\title{
Revisiting Evolutionary Game Theory
}

Ilaria Brunetti, Eitan Altman

\section{To cite this version:}

Ilaria Brunetti, Eitan Altman. Revisiting Evolutionary Game Theory. [Research Report] 2013. hal00801851

\section{HAL Id: hal-00801851 \\ https://hal.inria.fr/hal-00801851}

Submitted on 18 Mar 2013

HAL is a multi-disciplinary open access archive for the deposit and dissemination of scientific research documents, whether they are published or not. The documents may come from teaching and research institutions in France or abroad, or from public or private research centers.
L'archive ouverte pluridisciplinaire HAL, est destinée au dépôt et à la diffusion de documents scientifiques de niveau recherche, publiés ou non, émanant des établissements d'enseignement et de recherche français ou étrangers, des laboratoires publics ou privés. 


\title{
Revisiting Evolutionary Game Theory*
}

\author{
Ilaria Brunetti and Eitan Altman ${ }^{\dagger}$
}

\begin{abstract}
Evolutionary game theory is a relatively young mathematical theory that aims to formalize in mathematical terms evolution models in biology. In recent years this paradigm has penetrated more and more into other areas such as the linguistics, economics and engineering. The current theory of evolutionary game makes an implicit assumption that the evolution is driven by selfishness of individuals who interact with each others. In mathematical terms this can be stated as "an individual equals a player in a game model". This assumption turns out to be quite restrictive in modeling evolution in biology. It is now more and more accepted among biologist that the evolution is driven by the selfish interests of large groups of individuals; a group may correspond for example to a whole beehive or to an ants' nest. In this paper we propose an alternative paradigm for modeling evolution where a player does not necessarily represent an interacting individual but a whole class of such individuals.
\end{abstract}

\section{INTRODUCTION}

Evolutionary game theory (EGT) started as a theory that intended to explain and predict quantitative and qualitative aspects of evolution in biology by using the powerful tools of game theory [10]. It focused on pairwise interactions, assuming selfishness of individuals who interact. Strategies are interpreted as inheritable traits and payoff as Darwinian fitness. Standard EGT has some modeling weaknesses since it identifies each individual which interacts with an individual as a selfish player.

We found several examples that put this model in question:

- in some species like bees or ants, the one who interacts is not the one who reproduces. This implies that the Darwinian fitness is related to the entire swarm and not to a single bee.

- In many species, we find altruistic behaviors, which may hurt the individual adopting it, favoring instead the group he belongs to. Altruistic behaviors are typical of parents toward their children: they may incubate them, feed

*The research was financed by the Congnas Eureopean project

$\dagger$ The authors are with the Maestro team of INRIA, 2004, Route des Lucioles, 06902 Sophia Antipolis, France. Email: \{Ilaria.Brunetti, Eitan.Altman\} Qinria.fr them or protect them from predators at a high cost for themselves. Another example can be found in flock of birds: when a bird sees a predator it gives an "alarm call" to warn the rest of the flock, attracting the predator's attention to itself. Also the stringing behavior of bees is an altruistic one: it serves to protect the hive, but it's lethal for the bees which strives.

- In engineering applications: in wireless communication, power control games have frequently been studied in the framework of standard EGT. Papers that consider these games always assume that each mobile can control selfishly its power. In practice however the protocols for power control are not determined by the users of the terminal but by the equipment constructors; with us argues that the real competition is among a final number of equipment constructors.

In this paper we present a new model for evolutionary games, in which the concept of the player as a single individual is substituted by that of a player as a whole group of individuals. Even if we still consider pairwise interactions among individuals, our perspective is completely different: we suppose that individuals are simple actors of the game and that the utility to be maximized is the one of their group.

We analyze one of the most studied examples in evolutionary games, that of the Hawk and the Dove, which is a model for determining the degree of aggressiveness in the population. For this particular game we compute the symmetric Nash equilibrium and we show that the fact of teaming together makes individuals less aggressive at equilibrium.

The structure of the paper is as follows. In the next section we provide an overview and related literature. In section III we present the mathematical model of both the classical evolutionary game theory as well as our new definitions of group evolutionary game theory. We formulate games between several populations, both the case of infinite as well as finite populations are considered. We consider also the case in which the number of 
players is not known to the decision makers. In Section IV we present the Hawk and Dove game in GEG context.

\section{OVERVIEW OF RELATED APPROACHES}

Evolutionary game theory was introduced by John Meynard Smith [6], [7] to explain evolutionary processes in nature related to competition over resources. It is restricted to models of pairwise interactions between individuals. It assumes that an individual behaves as if it were a player, or a decision maker which maximizes its utility. The utility is taken to be the fitness of the individual; it is thus directly related to the reproduction rate of the individual. It is a function of both features (or behavior) of the individual as well as the distribution of features (or behavior types) among the whole population. Thus natural selection can be modeled as a game in which behaviors or features are actions, and the players are individuals.

When the number of players is very large, it is often well approximated by an infinite number of non-atomic players. This approach to model a large number of users have already been used in road traffic Engineering, where, however, an individual is not involved in pairwise interactions but in interactions with infinitely many individuals who share common roads. Such games are often called "population games". The equilibrium notion known as Wardrop equilibrium to those games was introduced already in 1952 [9]. We naturally find this equilibrium concept also in telecommunication networks when the number of decision makers is very large [1].

Much more recently, researchers in both road traffic [2] as well as in telecommunication networks [5] have identified situations in which a whole group of cars can be associated with a single decision maker. A particular attention was given to situations in which the number of such groups is finite, and each group contains a strictly positive fraction of the whole population. These are often called splittable atomic games. In road traffic, such a group may correspond to a transportation company. In telecommunication networks it may correspond to an Internet Service Provider that may control how its traffic will be routed in the network. The equilibrium concept for these games, that of Nash equilibrium, is different than the Wardrop equilibrium, but under suitable condition the former converges to the latter as the number of groups grows to infinity (see [2]).

We argue in this paper that the competition between groups should also be considered in the context of evolutionary games as it models many biological phenomena better than the standard evolutionary game paradigm. We shall show in an example how the Nash equilibrium obtained may indeed differ from that in which each individual is a player.

\section{MODEL AND NEW CONCEPTS}

\section{A. Classical Evolutionary Games}

Evolutionary game theory is based on a setting with the following main features:

1) There is a large population of individuals, so large that it can be represented as a "fluid of individuals".

2) These individuals interact with each other through a large number of pairwise interactions.

3) There is a finite set $\mathbf{A}$ of actions. An individual choosing an action $a$ facing another individual who chooses action $b$ receives a payoff $u(a, b)$.

4) If an individual chooses an action $a$ in a random way using a probability $\mathbf{p}$ (whose entries are $p(a), a \in \mathbf{A})$ and every other player chooses an action $b$ according to a probability $\mathbf{q}$ (whose entries are $q(b)$ ) then the expected payoff for the individual is

$$
u(p, q)=\mathbf{p}^{T} \mathbf{u q} \text {. }
$$

where $\mathbf{u}$ is the matrix whose $a, b$ entry is $u(a, b)$.

5) Each individual is a decision maker and he chooses an action so as to maximize his own expected fitness. $p$ is a Nash equilibrium if $u(p, p) \geq u(q, p)$ for all possible $q$.

In this paper we replace point (5) above by distinguishing among actors, that is the individuals who chooses the action, and players, that is the groups of individuals whose utility is maximized. The rules that determine the actions of individuals are chosen so as to maximize the utility of their group. Note that in biological models this utility will represent the Darwinian's fitness of the group, and the decision process is done by darwinian selection, related to the whole group. We shall consider in this paper a finite number of competing groups and we will hence use the Nash equilibrium as solution concept.

B. Our new model: infinite population of individuals with a finite number of players

We consider an infinite population of individuals, divided into $N$ groups; for simplicity of 
presentation we will consider symmetric groups of the same size. We can also think at a population composed of an higher number of groups, but in which each one of them only interacts with $N$ neighbors.

As in standard evolutionary games, we focus on pairwise interactions, where each actor has a finite set of available actions: $\mathbf{A}=\left\{a_{1}, a_{2} \ldots a_{M}\right\}$. We suppose that all individuals in the same group use the same strategy, so that the probability of choosing an action only depends on the group the individual belongs to. Given an actor $j$ in group $N_{i}$, the probability of choosing an action $a_{k} \in \mathbf{A}$ is:

$$
\mathbb{P}\left(a_{i, j}=a_{k}\right)=\mathbb{P}\left(a_{i}=a_{k}\right)=p_{i_{k}}
$$

$\forall i=1, \ldots, N \quad k=1 \ldots M$

We associate to each group $i$ the vector of probabilities $\bar{p}_{i}$ :

$$
\bar{p}_{i}=\left(p_{i_{1}}, p_{i_{2}}, \ldots, p_{i_{M}}\right)
$$

where $\sum_{l=1}^{M} p_{i_{l}}=1 \quad \forall i=1, \ldots, N$.

As the population is infinite,we can assume that the probability of an interaction among two individuals of the same group equals the probability of an interaction among actors of different groups.

Since an individual is equally likely to interact with an individual of any one of the of the $N$ groups (including its own one), the expected utility of a group (player) $i$ is:

$$
\mathbb{U}_{i}\left(\bar{p}_{i}, \bar{p}_{-i}\right)=\frac{1}{N} U_{\bar{p}_{i}, \bar{p}_{i}}+\frac{1}{N} \sum_{j=1, j \neq i}^{N} U_{\bar{p}_{i}, \bar{p}_{j}}
$$

where $U_{\bar{p}_{i} \bar{p}_{j}}\left(U_{\bar{p}_{i} \bar{p}_{i}}\right)$ is the immediate reward of an individual playing $\bar{p}_{i}$ against an opponent playing $\bar{p}_{j}\left(\bar{p}_{i}\right)$.

Since we model a finite set of players, the natural solution concept is the Nash equilibrium.

\section{The case of a finite population of individuals}

As in our models a player represents a group of individuals, it's important to study the impact of the size of groups on the equilibrium. We thus consider now a finite population divided into $N$ groups of size $K$.

Since the population is finite, given an actor in group $N_{i}$ and his opponent in $N_{j}, i, j \in$ $\{1, \ldots, N\}$, the probability that they belong to the same group is:

$$
\mathbf{P}(i=j)=\frac{K-1}{(K-1)+(N-1) K}=\frac{K-1}{N K-1}
$$

whereas the probability of belonging to two different groups is:

$$
\mathbf{P}(i \neq j)=1-\frac{K-1}{N K-1}=\frac{K(N-1)}{N K-1}
$$

The expected utility of a player $i$ is:

$$
\begin{aligned}
\mathbb{U}_{i}\left(\bar{p}_{i}, \bar{p}_{-i}\right) & =\frac{K-1}{N K-1} U_{\bar{p}_{i} \bar{p}_{i}}+ \\
& +\frac{K}{N K-1} \sum_{j=1, j \neq i}^{N} U_{\bar{p}_{i} \bar{p}_{j}}
\end{aligned}
$$

\section{The case of a random number of groups}

In order to represent all those situations in which the number of groups a player interacts with is not known, we define a third model in which $\mathrm{N}$ is random. More generally we want to consider the case in which the total number of groups is fixed, but each group $i$ interacts with a random number $N_{i}$ of players. We suppose that the population is finite and that the size of each group is $K$.

For each $i=1 \ldots N$ we suppose that $N_{i}$ follows a geometric distribution:

$$
\mathbb{P}\left(N_{i}=n\right)=(1-q)^{n-1} q
$$

We assume that all the players but a fixed $i$ use the same strategy, so that $\forall j \neq i \quad p_{j}=p$.

The average utility for the player $i$ is:

$$
\begin{aligned}
& \mathbb{U}\left(\bar{p}_{i}, \bar{p}\right)=\sum_{n=1}^{\infty} q(1-q)^{n-1} \times \\
& \times\left[\frac{K-1}{n K-1} U_{\bar{p}_{i} \bar{p}_{i}}+\frac{K(n-1)}{n K-1} U_{\bar{p}_{i} \bar{p}_{j}}\right]
\end{aligned}
$$

\section{HAWK AND Dove GAME IN GEGT}

\section{A. Hawk and Dove game in standard EGT}

The Hawk and Dove game has been first introduced by Maynard Smith and Price in The Logic of Animal Conflict to describe animal behavior [3]; the model has been one of the most studied problems in EGT, and it has been applied to many different contexts. This basic model allows to study the evolution of aggressiveness within a population.

It was used not only in biology but also in Engineering in order to study the impact of selfishness on the choice of protocols for both congestion control, access control and power control [8].

Hawk and Dove game represent a competition between two individuals for a resource. Each individual dispose of two possible strategies: he can 
choose to be a "hawk" and fight or he can choose to be a "dove" and peacefully back down. When two hawks meet, there will always be a fight, where the winner receive the benefit and the loser is injured. Doves are never aggressive, and so they are never involved in a fight. There is no cost to be a dove, only the possibility of receiving no payoff.

In order to illustrate our GEGT model, we suppose that any pairwise interaction is an Hawk and Dove game.

The set of available actions for each actor is $\mathbf{A}=\{H, D\}$, where $\mathrm{H}$ corresponds to playing aggressively and D not aggressively. We associate to each group the probability

$$
p_{i}=\mathbb{P}\left(a_{i}=H\right) \quad \forall i=1, \ldots, N
$$

We define the following payoff matrix for the Hawk and Dove game:

\begin{tabular}{|c|c|c|}
\hline & $H$ & $D$ \\
\hline$H$ & $-\delta+\frac{1}{2}$ & 1 \\
\hline$D$ & 0 & $\frac{1}{2}$ \\
\hline
\end{tabular}

where $\delta>\frac{1}{2}$

This allows us to compute the symmetric Nash equilibrium for our model, for the three cases we have presented in the previous sections.

\section{B. Infinite population of individuals}

In the case of an infinite population, we can rewrite the utility of a player $i$ defined in (2) as:

$$
\begin{aligned}
& \mathbb{U}_{i}\left(p_{i}, p_{-i}\right)=\frac{1}{N} U_{p_{i}, p_{i}}+\frac{1}{N} \sum_{j=1, j \neq i}^{N} U_{p_{i}, p_{j}} \\
& =\frac{1}{N}\left[(-\delta) p_{i}^{2}+\frac{1}{2}\right]+ \\
& +\frac{1}{N} \sum_{j=1, j \neq i}^{N}\left[\left(-\delta p_{j}+\frac{1}{2}\right) p_{i}+\frac{1-p_{j}}{2}\right]
\end{aligned}
$$

We seek for the symmetric Nash equilibrium so we maximize the utility function :

$$
\begin{aligned}
& \frac{\partial \mathbb{U}_{i}\left(p_{i}, p_{-i}\right)}{\partial p_{i}}=\frac{-2 \delta}{N} p_{i}+\frac{1}{N} \sum_{j=1, j \neq i}^{N}\left(-\delta p_{j}+\frac{1}{2}\right) \\
& =\frac{2}{N}(-\delta) p_{i}+\frac{1}{N} \sum_{j=1, j \neq i}^{N}\left(\frac{1}{2}-\delta p_{j}\right)
\end{aligned}
$$

We impose $\frac{\partial \mathbb{U}_{i}\left(p_{i}, p_{-i}\right)}{\partial p_{i}}=0$ :

$$
p_{i}=\frac{1}{2 \delta N} \sum_{j=1, j \neq i}^{N}\left(-\delta p_{j}+\frac{1}{2}\right)
$$

By imposing $p_{i}=p_{j}=p$, we obtain the symmetric Nash equilibrium of the game:

$$
p=\frac{N-1}{N+1} \frac{1}{2 \delta}
$$

It is interesting to study two extreme cases:

- $N \rightarrow \infty$ When the number of groups is infinite we obtain:

$$
p=\frac{1}{2 \delta}
$$

which is the value of the equilibrium of the corresponding standard evolutionary games.

This is consistent with a similar result in [2], that shows the convergence of Nash equilibrium to Wardrop equilibrium as the number of players goes to infinity.

- $N=2$ When we have only two players formed by an infinity of actors, we obtain that

$$
p=\frac{1}{6 \delta}
$$

which means that, as expected, two groups are less aggressive then two standard players.

Figure 1 shows the equilibrium probability of being aggressive $p$ as a function of the number of players $N$. We can observe that the equilibrium $p$ is an increasing function in the number of groups. Note that when $N$ increases, the probability of meeting a member of a different group increases: so we found that the level of aggressiveness is higher when the probability of interactions among individuals of different groups increases. Hence if an individual is aggressive it causes less damage to his group. This can explain the fact that the equilibrium probability of being aggressive increases in $N$.

As one may expect, we also observe that when the $\operatorname{cost} \delta$ (involved in an encounter between two aggressive individuals) increases, the equilibrium probability $p$ of being aggressive decreases.

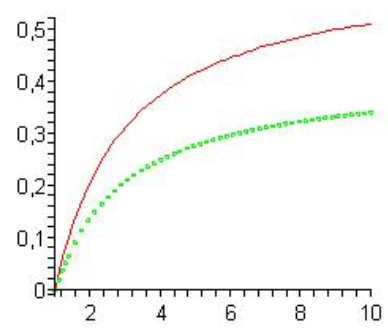

Fig. 1. The value of $\mathrm{p}$ as a function of the number of groups $\mathrm{N}$ for two different values of $\delta$. The continuous line (the upper curve) is obtained with $\delta=0.8$, the dotted line (lower curve) with $\delta=1.2$ 


\section{Finite population of individuals}

We now consider the second case, that of a finite population of individuals. When considering the payoff matrix (5), the utility defined in (3) becomes:

$$
\begin{aligned}
& \mathbb{U}_{i}\left(p_{i}, p_{-i}\right)=\frac{K-1}{N K-1}\left[(-\delta) p_{i}^{2}+\frac{1}{2}\right]+ \\
& +\frac{K}{N K-1} \sum_{j=1, j \neq i}^{N}\left[\left(-\delta p_{j}+\frac{1}{2}\right) p_{i}+\frac{1-p_{j}}{2}\right]
\end{aligned}
$$

As in the previous case, in order to maximize the utility we compute:

$$
\begin{gathered}
\frac{\partial \mathbb{U}_{i}\left(p_{i}, p_{-i}\right)}{\partial p_{i}}= \\
=2 \frac{K-1}{N K-1}(-\delta) p_{i}+\frac{K}{N K-1} \sum_{j=1, j \neq i}^{N}\left(-\delta p_{j}+\frac{1}{2}\right) \\
=2 \frac{K-1}{N K-1}(-\delta) p_{i}+\frac{K}{N K-1} \sum_{j=1, j \neq i}^{N}\left(\frac{1}{2}-\delta p_{j}\right)
\end{gathered}
$$

and we impose $\frac{\partial \mathbb{U}_{i}\left(p_{i}, p_{-i}\right)}{\partial p_{i}}=0$. We obtain:

$$
p_{i}=\frac{K}{2(K-1)} \sum_{j=1, j \neq i}^{N}\left(-p_{j}+\frac{1}{2 \delta}\right)
$$

and by imposing the symmetry:

$$
p=\frac{K(N-1)}{2(K-1)}\left(\frac{1}{2 \delta}-p\right)
$$

We finally obtain the following Nash equilibrium:

$$
p=\frac{K(N-1)}{K(1+N)-2} \frac{1}{2 \delta}
$$

In Figure 2 we plotted the value of the equilibrium $p$ as a function of $N$ for two different values of $K$ and a fixed $\delta$. As in the previous case, we can observe that $p$ is an increasing function of $\mathrm{N}$. In Figure (3) we plotted $p$ as a function of the size of the groups. We can note that $p$ rapidly decreases for small $K$; when $K>10, p$ stabilizes and it is very slowly decreasing. The explanation for this behavior is that when $K$ is small, then the probability of meeting an individual of one's own group is quite sensitive to $K$, which is not the case when $K$ is large.

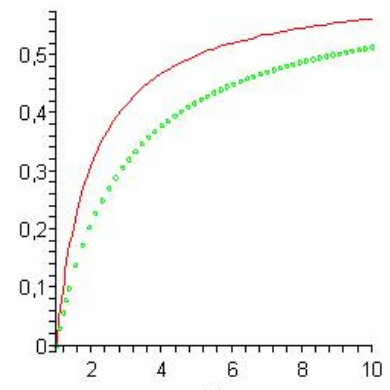

Fig. 2. The value of $\mathrm{p}$ as a function of the number of groups $\mathrm{N}$ for two different values of $K$ and $\delta=0.8$. Continuous line is obtained with $K=2$, dotted line with $K=50$

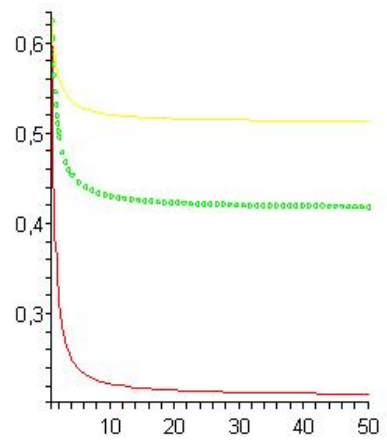

Fig. 3. The value of $\mathrm{p}$ as a function of the size of groups $\mathrm{K}$ for three different values of $N$.Lower continuous line is obtained with $N=2$, green line with $N=5$, higher continuous line with $N=10$.

\section{Random number of groups}

In the third case, that of a finite population divided into a random number of groups, the utility function (4) when considering the payoff matrix (5) becomes

$$
\begin{aligned}
& \mathbb{U}\left(p_{i}, p\right)=\frac{q\left((1-2 \delta p) p_{i}+1-p\right)}{2} \sum_{n=1}^{\infty}(1-q)^{n-1}+ \\
& +q \frac{K-1}{2}\left(-2 \delta p_{i}^{2}-(1-2 \delta p) p_{i}+p\right) \times \\
& \times \sum_{n=1}^{\infty} \frac{(1-q)^{n-1}}{n K-1}
\end{aligned}
$$

See derivation in Fig. VII in the Appendix VII. The $\operatorname{sum} \sum_{n=1}^{\infty}(1-q)^{n-1}=\sum_{n=0}^{\infty}(1-q)^{n}$ is a geometric series which converges to $\frac{1}{q}$. We define $A \equiv \sum_{n=1}^{\infty}(1-q)^{n-1} \frac{1}{n K-1}$ and we obtain:

$$
\begin{aligned}
& \mathbb{U}\left(p_{i}, p\right)=\frac{\left((1-2 \delta p) p_{i}+1-p\right)}{2}+ \\
& +q \frac{K-1}{2}\left(-2 \delta p_{i}^{2}-(1-2 \delta p) p_{i}+p\right) A
\end{aligned}
$$


As we want to optimize the expected utility, we impose $\frac{\partial \mathbb{U}\left(p_{i}, p\right)}{\partial p_{i}}=0$ :

$$
\frac{1-2 \delta p}{2}+q \frac{K-1}{2}\left(-4 \delta p_{i}-1+2 \delta p\right) A=0
$$

By imposing $p_{i}=p$, we have:

$$
2 \delta(q(K-1) A+1) p=1-q(K-1) A
$$

And we find the symmetric Nash equilibrium:

$$
\begin{aligned}
p & =\frac{1}{2 \delta} \frac{1-q(K-1) A}{1+(q(K-1) A} \\
& =\frac{1}{2 \delta}\left(\frac{2}{1+(q(K-1) A}-1\right)
\end{aligned}
$$

Figure 4 plots the equilibrium probability of being aggressive $p$ as a function of $q$ for three different values of $K$ and a fixed $\delta$. Taking into account that $q=1 / E[N]$, the form of the graph is indeed as can be expected from Figure 1 and Figure 2 in which the case of deterministic $N$ was considered (note however that the two latter graphs is depicted as a function of $N$ and not of $1 / N$ ).

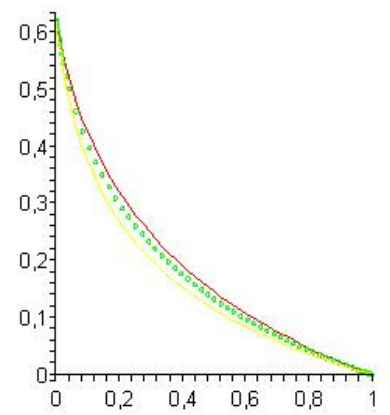

Fig. 4. The value of $\mathrm{p}$ as a function of $q$, for different values of $K$ and $\delta=0.8$. Continuous line on the right is obtained with $K=5$, dotted line with $K=25$ and continuous left one with $K=50$

Figure 5 shows the equilibrium $p$ as a function of $K$ for three different values of $N$. The form of the graph is analogous to the one observed in Figure 3.

\section{Concluding Section And Discussion}

This paper proposes a new alternative way to model evolutionary games in which fitness can be associated to a whole group rather than to an individual. Since fitness is defined in evolutionary games as related to the reproduction rate, the classical evolutionary game paradigm cannot model situations in which only one selected member of a

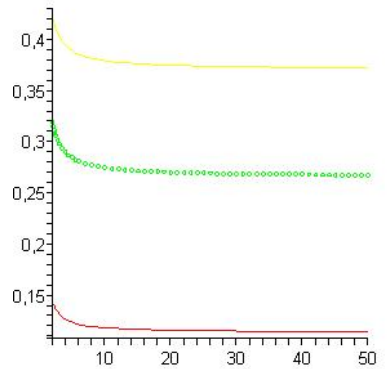

Fig. 5. The value of $\mathrm{p}$ as a function of the size of groups $\mathrm{K}$ for three different values of $q$. Lower continuous line is obtained with $q=0.5$, dotted line with $q=0.2$, higher continuous line with $q=0.1$

group is responsible for reproduction (beehives or nests of ants). We thus face a situation in which the decision maker (player) is not the individual involved in the interactions. The player can be identified with the group as a whole. We believe that our framework is useful also for other cases in which altruism is observed in nature between members of a family or of a larger group.

We considered here a finite number of players interacting with each other through pairwise interactions of their corresponding population. The equilibrium concept considered was naturally the Nash equilibrium which is a multistrategy such that no group can profit by deviating. In standard evolutionary games one often uses the notion of Evolutionary Stable Strategy (ESS) as a solution concept, which is stronger than a Nash equilibrium (it is robust against deviation of a whole small fraction of mutations). In our new GEG paradigm, Nash equilibrium implies robustness not just against a fraction of mutations in a group but against deviations of the whole group. Therefore, if we were to define an equilibrium in our model as a multistrategy robust against a small fraction of mutations, then it will not be a stronger equilibrium notion than the Nash equilibrium, as is the case in standard EG, but instead, any Nash equilibrium will also satisfy this definition of equilibrium.

\section{REFERENCES}

[1] E. Altman and L. Wynter. Equilibrium, games, and pricing in transportation and telecommunications networks. Networks and Spatial Economics, 4(1):7-21, March 2004 special issue on "Crossovers between Transportation Planning and Telecommunications".

[2] A. Haurie and P. Marcotte. On the relationship between nash-cournot and wardrop equilibria. Networks, 15:295308, 1985.

[3] j. Maynard Smith and G. R. Price. The logic of animal conflict. Nature, 246:15 - 18, 02 November 1973. 
[4] Luis M Navas, Francisco J. Ruiz, and Juan I. Varona. Asymptotic behavior of the lerh transcedent function. Journal of Approximation Theory, 2012.

[5] A. Orda, R. Rom, and N. Shimkin. Competitive routing in multi-user communication networks. IEEE/ACM Transactions on Networking, 1(5):510-520, 1993.

[6] J. Maynard Smith. Game theory and the evolution of fighting. J. Maynard Smith, On Evolution (Edinburgh: Edinburgh University Press), pages 8-28, 1972.

[7] J. Maynard Smith. Evolution and the theory of Games. Cambridge University Press, UK, 1982.

[8] Hamidou Tembine, Eitan Altman, Rachid El Azouzi, and Yezekael Hayel. Evolutionary games in wireless networks. IEEE Transactions on Systems, Man, and Cybernetics, Part B, 40(3):634-646, 2010.

[9] J. G. Wardrop. Some theoretical aspects of road traffic research communication networks. Proc. Inst. Civ. Eng., Part 2, 1:325-378, 1952.

[10] J. Weibull. Evolutionary Game Theory. M.I.T. Press, 1995

\section{Appendix: Evaluating A}

We want to evaluate the sum

$$
A \equiv f(1-q)=\sum_{n=1}^{\infty}(1-q)^{n-1} \frac{1}{n K-1}
$$

We define

$$
g(\alpha)=\sum_{n=1}^{\infty} \alpha^{n+r}=\alpha^{r+1} \sum_{n=0}^{\infty} \alpha^{n}=\frac{\alpha^{r+1}}{1-\alpha}
$$

where $0<\alpha<1$. From Fubini's theorem:

$$
\begin{aligned}
& \int g(\alpha) d \alpha=\int \sum_{n=1}^{\infty} \alpha^{n+r} d \alpha \\
& =\sum_{n=1}^{\infty} \int \alpha^{n+r} d \alpha=\sum_{n=1}^{\infty} \frac{\alpha^{n+r+1}}{n+r+1}
\end{aligned}
$$

From the equality (9) we can also write:

$$
\begin{aligned}
& \int g(\alpha)=\int \frac{\alpha^{r+1}}{1-\alpha} \\
& =\alpha^{r+1}\left(-\frac{1}{r+1}+\operatorname{LerchPhi}(\alpha, 1, r+1)\right)
\end{aligned}
$$

where LerchPhi is the Lerch Zeta function defined as [4]:

$$
\operatorname{LerchPhi}(z, a, v) \equiv \sum_{n=0}^{\infty} \frac{z^{n}}{(v+n)^{a}}
$$

We thus obtain that:

$$
\sum_{n=1}^{\infty} \frac{\alpha^{n+q}}{n+q}=\alpha^{q}\left(-\frac{1}{q}+\operatorname{LerchPhi}(\alpha, 1, q)\right)
$$

where $q=r+1$.

We now rewrite $f(\alpha)$ :

$$
\begin{aligned}
& f(\alpha)=\sum_{n=1}^{\infty}(\alpha)^{n-1} \frac{1}{n K-1}=\frac{1}{\alpha K} \\
& =\sum_{n=1}^{\infty} \frac{\alpha^{n}}{n-\frac{1}{K}}=\frac{\alpha^{-1+\frac{1}{K}}}{K} \sum_{n=1}^{\infty} \frac{\alpha^{n-\frac{1}{K}}}{n-\frac{1}{K}}
\end{aligned}
$$

By considering $q=-\frac{1}{K}$, we get the following expression for $f(\alpha)$ :

$$
\begin{aligned}
& \frac{\alpha^{-1+\frac{1}{K}}}{K}\left(\alpha^{-\frac{1}{K}}\left(-\frac{1}{-\frac{1}{K}}+\operatorname{LerchPhi}\left(\alpha, 1,-\frac{1}{K}\right)\right)\right) \\
& =\frac{1}{\alpha K}\left(-K+\operatorname{LerchPhi}\left(\alpha, 1,-\frac{1}{K}\right)\right)
\end{aligned}
$$

\section{APPENDIX: DERIVATION OF THE UTILITY} FOR A RANDOM NUMBER OF PLAYERS

The derivation is provided in Figure VII. 


$$
\begin{aligned}
\mathbb{U}\left(p_{i}, p\right) & =\sum_{n=1}^{\infty} q(1-q)^{n-1}\left[\frac{K-1}{n K-1}\left(-\delta p_{i}^{2}+\frac{1}{2}\right)+\frac{K(n-1)}{n K-1}\left(\left(\frac{1}{2}-\delta p\right) p_{i}+\frac{1-p}{2}\right)\right] \\
& =\sum_{n=1}^{\infty} q(1-q)^{n-1}\left[\frac{(K-1)\left(-\delta p_{i}^{2}+1\right)}{2(n K-1)}+\frac{(K(n-1)+1-1)\left((1-2 \delta p) p_{i}+1-p\right)}{2(n K-1)}\right] \\
& =\sum_{n=1}^{\infty} q(1-q)^{n-1}\left[\frac{(K-1)\left(-\delta p_{i}^{2}+1\right)}{2(n K-1)}-\frac{(K-1)\left((1-2 \delta p) p_{i}+1-p\right)}{2(n K-1)}+\frac{(1-2 \delta p) p_{i}+1-p}{2}\right] \\
& =\sum_{n=1}^{\infty} q(1-q)^{n-1}\left[\frac{(K-1)\left(-\delta p_{i}^{2}+p-(1-2 \delta p) p_{i}\right)}{2(n K-1)}+\frac{(1-2 \delta p) p_{i}+1-p}{2}\right] \\
& =\frac{q\left((1-2 \delta p) p_{i}+1-p\right)}{2} \sum_{n=1}^{\infty}(1-q)^{n-1}+q \frac{K-1}{2}\left(-2 \delta p_{i}^{2}-(1-2 \delta p) p_{i}+p\right) \sum_{n=1}^{\infty} \frac{(1-q)^{n-1}}{n K-1}
\end{aligned}
$$

Fig. 6. Computing the expected utilities when the number of players is random 